\title{
Optical trapping microrheology in cultured human cells ${ }^{\star}$
}

\author{
E. Bertseva ${ }^{1, a}$, D. Grebenkov ${ }^{2,3}$, P. Schmidhauser ${ }^{1}$, S. Gribkova ${ }^{2}$, S. Jeney ${ }^{1,4}$, and L. Forró ${ }^{1}$ \\ ${ }^{1}$ Laboratory of Physics of Complex Matter, Ecole polytechnique federale de Lausanne (EPFL), Station 3, CH-1015 Lausanne \\ VD, Switzerland \\ 2 Laboratoire de Physique de la Matière Condensée, CNRS - Ecole Polytechnique, F-91128 Palaiseau, France \\ 3 Laboratoire Poncelet (UMI 2615), CNRS - Independent University of Moscow, Bolshoy Vlasyevskiy Pereulok 11, 119002 \\ Moscow, Russia \\ 4 M E Müller Institute for Structural Biology, Biozentrum, University of Basel, Basel, Switzerland
}

Received 18 July 2011 and Received in final form 8 November 2011

Published online: 23 July 2012 - (C) EDP Sciences / Società Italiana di Fisica / Springer-Verlag 2012

\begin{abstract}
We present the microrheological study of the two close human epithelial cell lines: non-cancerous HCV29 and cancerous T24. The optical tweezers tracking was applied to extract the several seconds long trajectories of endogenous lipid granules at time step of $1 \mu \mathrm{s}$. They were analyzed using a recently proposed equation for mean square displacement (MSD) in the case of subdiffusion influenced by an optical trap. This equation leads to an explicit form for viscoelastic moduli. The moduli of the two cell lines were found to be the same within the experimental accuracy for frequencies $10^{2}-10^{5} \mathrm{~Hz}$. For both cell lines subdiffusion was observed with the exponent close to $3 / 4$, the value predicted by the theory of semiflexible polymers. For times longer than $0.1 \mathrm{~s}$ the MSD of cancerous cells exceeds the MSD of non-cancerous cells for all values of the trapping force. Such behavior can be interpreted as a signature of the active processes and prevents the extraction of the low-frequency viscoelastic moduli for the living cells by passive microrheology.
\end{abstract}

\section{Introduction}

Microrheology based on optical tweezers (OT) has become a rapidly growing field in recent years (for review see refs. [1-3] and references therein). In passive microrheology a probe particle thermally fluctuates inside the substance under study, and the mean square displacement (MSD) of its positions allows one to extract physical quantities such as stiffness [3], compliance [4], viscosities [5] or dynamic moduli $[4,6,7]$. In active microrheology the particle exerts a force on the sample. The examples are the magnetic tweezers $[8,9]$, oscillating OT [10-12] and the membrane tether pulling techniques [13].

Changes in cell mechanics due to different types of cancer have been observed by many researchers (see review ref. [14]). Generally, cancer cells become softer than their normal analogues facilitating their motility and hence the metastatic processes. Most of the available studies have been performed by external methods such as stretching, squeezing, indentation, or flow essays. In this work, the passive microrheology provides the dynamical moduli in-

\footnotetext{
* Supplementary material in the form of a pdf file available from the Journal web page at

http://dx.doi.org/10.1140/epje/i2012-12063-4

${ }^{a}$ e-mail: elena.bertseva@epfl.ch
}

side a living cell by tracking endogenous granules which move in the cytoskeleton on a short length scale.

The cytoskeleton is a protein structure which plays a major mechanical role in the cell, maintaining the cell shape, protecting the cell organelles, enabling cellular motion and intracellular transport. It is a network of polymer filaments which belong to three main groups: microtubules, actin filaments and intermediate filaments [15, 16]. The biological function of these polymers requires considerable mechanical rigidity. For example, actin filaments form a network rigid enough to maintain the shape of the cell and transmit forces, yet flexible enough to allow for cell motion and internal reorganization in response to external stimuli. In vitro studies have revealed the mechanical properties of the individual filaments, as well as the properties of their networks in solution $[6,17]$.

Most biopolymers are semiflexible, i.e. the aspect ratio between the persistence length and the filament diameter is large. One of the distinct properties of semiflexible polymers is that they can form viscoelastic gels at very low concentrations. At high frequencies, the complex shear modulus of such gels presents a characteristic frequency dependence $G \sim \omega^{3 / 4}$ which has been explained in the model of semi-flexible polymer networks $[18,19]$. This model describes the dynamics of the in vitro solutions but fails for the cytoskeleton in vivo at frequencies below $10^{2}-10^{3} \mathrm{~Hz}$ [18]. It has been shown that at low frequencies 
the important role is played by the network cross-linkers, in particular by the molecular motors [20-22]. Varying the cross-linker density at a constant concentration of polymers can change the low frequency shear modulus by several orders of magnitude [23]. But at high frequencies the classical, $3 / 4$, behavior of the semi-flexible polymers is recovered both in vitro and in vivo $[9,20,24-26]$. The frequency of the transition between the two types of response, therefore, is expected to be connected to the type and strength of cross-linking and motor activity inside the cytoskeleton.

The viscoelastic moduli for the living cells obtained by particle-tracking microrheology have been recently summarized in ref. [3], but this technique provides the results only at frequencies $0.1-10^{2} \mathrm{~Hz}$, corresponding to video detection. To our knowledge, there exist only a limited number of works with the high-frequency microrheology for the cytoskeleton of the living cells [9,20,24-27], and only one of them presents the full frequency range of viscoelastic moduli [24]. We use the optical tweezers with rather strong trapping powers to hold the tracer for longer time and to obtain several-seconds-long samples at $1 \mathrm{MHz}$ acquisition rate (millions of points per trajectory). The overall displacement of the tracer during the recording is ten times smaller than its diameter that makes a principal difference with the video tracking experiments [3], and provides the larger statistics for each MSD calculation.

In this work we perform the microrheological measurements by laser-tracking of the organelles trapped by the OT in the cytoskeleton of two related types of cells: the non-cancerous HCV29 and cancerous T24 epithelial cells of human urine bladder. These cells types have been chosen because the former elasticity measurements performed for them by Atomic Force Microscopy (AFM) [28, 29] have shown a higher elasticity for the cancerous T24 line. Young's modulus for the cancerous cell line was found to be about one order of magnitude lower than for noncancerous ones. However, the AFM differs from microrheology in two major ways: it uses the external probe and is conducted in a quasi-static regime which corresponds to the low frequencies for the OT microrheology.

We calculate the viscoelastic moduli $G^{\prime}$ and $G^{\prime \prime}$ for cancerous and non-cancerous cells and compare them for the two cell lines in the range of 4 decades of frequency: 10-10 $0^{5} \mathrm{~Hz}$. The OT exert their own elastic force on the tracer, which influences the MSD at low frequencies and complicates the calculation of viscoelastic moduli. We use the newly proposed theoretical approach $[30,31]$ to account for the tweezer-based elasticity in the analysis of the data. To test the validity of this approach we performed the measurements at different forces of the OT.

\section{Materials and methods}

The set-up consists of an optical trap combined with an interferometric position detection system. The system is described in details in refs. $[32,33]$. It is based on a Nd:YAG laser, which has a wavelength of $1064 \mathrm{~nm}$ and maximum power of $2 \mathrm{~W}$ (MEPHISTO Innolight, GmbH). The optical trap is composed of $10 \times$ beam expander and the
$60 \times$ water immersion objective (Olympus UPlanApo/IR, $\mathrm{NA}=1.2$ ).

The tracer trajectory is recorded through the back focal plane detection - the method which relies on the interference between forward-scattered light from the bead and unscattered light [34-36]. The interference signal is monitored with a quadrant photodiode positioned on the optical axis at a plane conjugate to the back focal plane of the condenser [37]. By performing a statistical analysis (explained in sect. 3) on the trajectories arising from the thermal fluctuations of the tracer and its close environment, we extract physical quantities and can follow their changes [3].

The cell lines chosen for the measurements are: HCV29, non-cancerous bladder urothelium (cell line established in Fibiger Institute, Copenhagen, Denmark), and T24, bladder transitional cell carcinoma (ATCC HTB4). The cells have been cultured at $37^{\circ} \mathrm{C}$ in a $95 \%$ air $/ 5 \% \mathrm{CO}_{2}$ incubator in RPMI medium containing $10 \%$ fetal bovine serum. The cells were taken after the same number of passages and cultured on standard glass coverslips. For the OT measurements the coverslips with cultured cells were placed on top of the microscope slides, separated by the spacers and the obtained chamber was filled with the solution of polysterene calibration beads of diameter $0.4 \mu \mathrm{m}$ in pure RPMI medium.

The cells contain the endogenous granules composed of lipids with sizes varying from 0.2 to $3 \mu \mathrm{m}$ (fig. 1 ). The granules of diameter about $0.4 \mu \mathrm{m}$ have been chosen as the microrheological tracers. For each slide we first trap the polystyrene beads outside the cell to calibrate the detector and to calculate the trap spring constant $k$. Then we trapped the endogenous granules of a similar diameter and recorded their trajectories using the calibration obtained with the beads. In order not to disturb the back focal plane detection, only the granules in the peripheral region of the cell and clearly separated from all other structures were chosen. The optical microscope does not allow us to choose exactly the same diameter, so the size has been verified through the post-experiment image analysis (see table 1SM in supplementary materials (SM)).

Three different spring constants of the applied optical trap: $k_{1}=4 \cdot 10^{-6} \mathrm{~N} / \mathrm{m}, k_{2}=10^{-5} \mathrm{~N} / \mathrm{m}, k_{3}=3 \cdot 10^{-5} \mathrm{~N} / \mathrm{m}$ correspond, respectively, to three laser powers in the sample plane: $5 \mathrm{~mW}, 10 \mathrm{~mW}, 40 \mathrm{~mW}$. At such powers and taking into account the weakly absorbing laser wavelength, we consider the temperature effects negligible.

The living cells are the highly dynamic system, and the granules are subject to both the passive fluctuations due to the surrounding network and the active drive due to the energy-consuming molecular motors. The chosen granule has been attracted to the center of the trap, and the measurement was started several seconds later, after the granule was stabilized in the trap. Nevertheless, some granules managed to escape the trap during the recording, and such trajectories have been excluded from the analysis. Also, we have excluded all data, containing the excessive displacement from the center of the trap - exceeding the linear region of detector. We have observed that the number of 


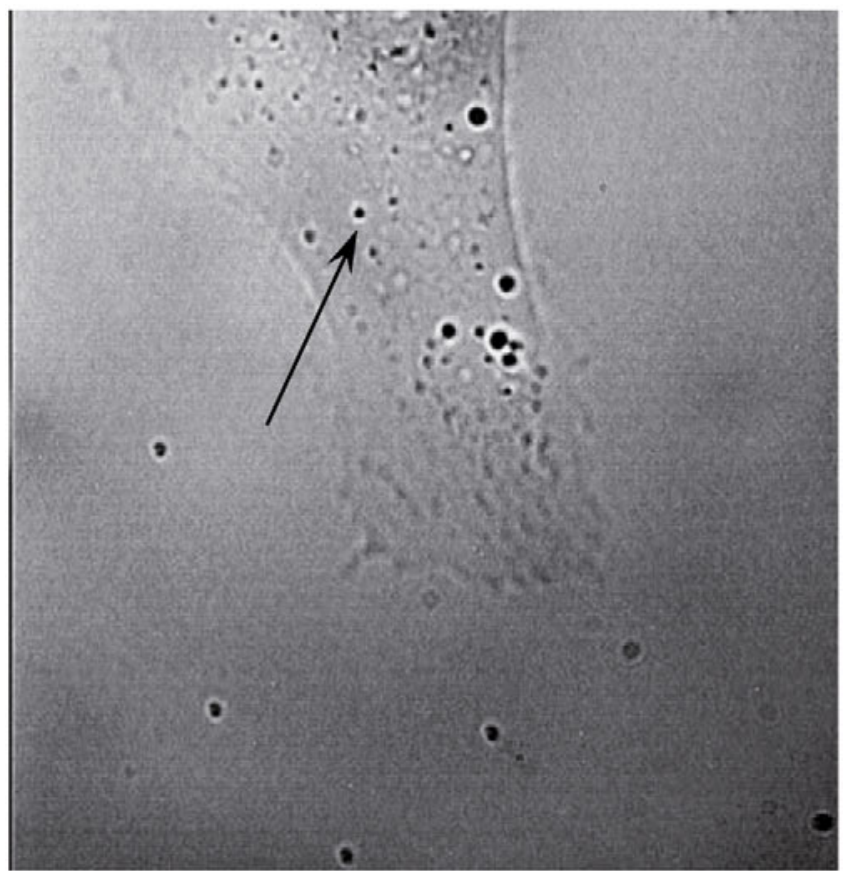

Fig. 1. HCV29 cell during the microrheology measurement. The arrow shows the position of the trapped lipid granule. The $0.4 \mu \mathrm{m}$ calibration beads stuck on the chamber surface are out of focus (and distorted with astigmatism) as well as the cell edges adherent to the substrate.

trajectories excluded for the T24 cell line exceeded that for HCV29, so the lipid granules in the cancerous cell line escaped the trap easier than in the non-cancerous one. It is difficult to quantify the number of escapes, because one cannot account for the measurements which had been interrupted due to escape of the tracer from the trap. But the number of granule escapes leading to the interruption of recoding has been higher for T24 cells, and the percentage of trajectories excluded from analysis because of nonlinearity was $50 \%$ for T24 against $30 \%$ for HCV 29 . The total number of trajectories chosen for the analysis was 33 for each cell line with a duration of 8 seconds each.

After the initial calibration using the detector constant obtained from the polysterene beads, the time-averaged MSD is calculated separately for each granule as

$$
\begin{aligned}
\Delta r(t)^{2}= & \frac{1}{N-t / \delta t} \sum_{i=1}^{N-t / \delta t}\left[(x(i \delta t+t)-x(i \delta t))^{2}\right. \\
& \left.+(y(i \delta t+t)-y(i \delta t))^{2}\right]
\end{aligned}
$$

where $x(t)$ and $y(t)$ are the coordinates of the granule at time $t, N$ is the number of points of a trajectory, $t$ is the lag time, and $\delta t=10^{-6} \mathrm{~s}$ is the time step of the recording. The background noise is evaluated by recording the signal from the empty trap placed inside the cell at a point without apparent structures. The MSDs calculated from such signal are averaged over several measurements and the result is subtracted from the tracer MSD for each measurement (see figs. 1SM-3SM).

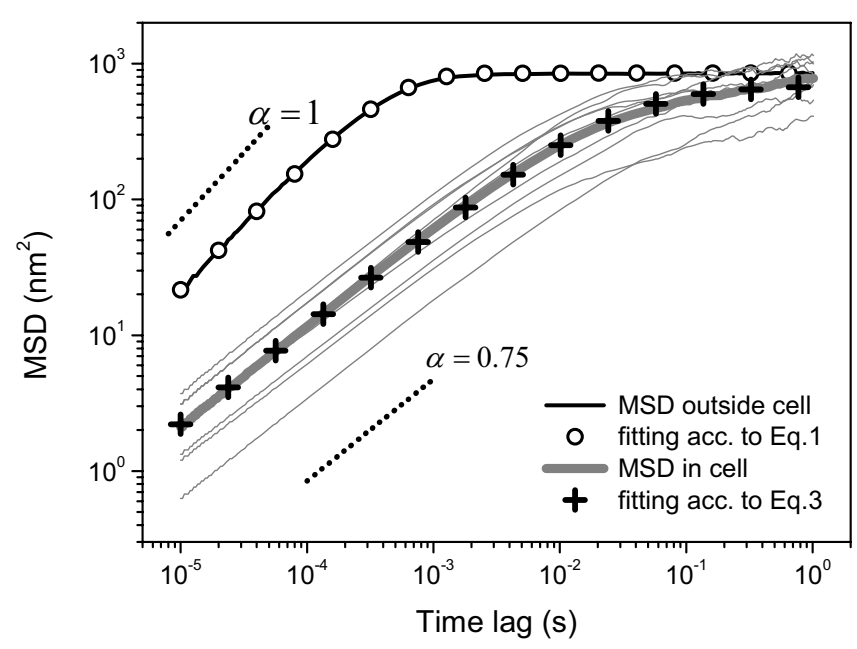

Fig. 2. The time-averaged mean-square displacement (MSD) of the $0.4 \mu \mathrm{m}$ diameter tracer in the purely viscous medium outside the cell (black line) and inside the HCV29 cell (thick grey line) where it presents the subdiffusive scaling. Thin grey lines show the MSDs of different granules inside the cells.

\section{Theoretical model}

Since the tracer is in contact with the microscopic environment, the structure of the surrounding medium influences the properties of the random motion and leaves its "signature" in the recorded signal. For example, it has been shown that the MSD of a tracer inside a viscoelastic medium does not follow Einstein's law, $\left\langle\Delta r(t)^{2}\right\rangle \sim t$, but presents the so-called subdiffusion scaling $\left\langle\Delta r(t)^{2}\right\rangle \sim t^{\alpha}$, where $\alpha<1$ is called the subdiffusion exponent $[22,38]$ (here $\langle\ldots\rangle$ denotes the ensemble average over all possible trajectories).

Figure 2 presents typical time-averaged MSDs: one obtained in the purely viscous medium outside the cell and the others in the viscoelastic interior of the cell. The thick curve is the average over ten MSDs for granules inside different cells shown by the thin lines.

The MSD curve for the bead outside the cell scales $\sim t$ at short times and reaches a constant due to the trap confinement at longer times. For an $n$-dimensional isotropic medium, this curve is analytically described as [39]

$$
\left\langle\Delta r^{2}(t)\right\rangle=\frac{2 k_{B} T}{k} n\left(1-\exp \left(-\frac{k}{\gamma} t\right)\right),
$$

where $k_{B}$ is Boltzmann's constant, $T$ the absolute temperature, $\gamma$ the friction coefficient, $k$ the trap spring constant, and $n=2$ in our case. For a spherical tracer of radius $a$, the friction coefficient is $\gamma=6 \pi a \eta$, where $\eta$ is the fluid viscosity. At short times, one retrieves $\left\langle\Delta r^{2}(t)\right\rangle \approx 2 n D t$, with the diffusion coefficient $D=k_{B} T / \gamma$.

We assume that the motion of a tracer inside the cell can be described by a generalized Langevin equation,

$$
m \ddot{x}+\int_{0}^{t} \mathrm{~d} t^{\prime} \gamma\left(t-t^{\prime}\right) \dot{x}\left(t^{\prime}\right)+k x=F(t),
$$

with a power law memory kernel 


$$
\gamma(t)=\gamma_{\alpha} \frac{t^{-\alpha}}{\Gamma(1-\alpha)},
$$

$\Gamma(z)$ being the Gamma function, $m$ the mass of the tracer and $F(t)$ the thermal Gaussian force with zero mean and the covariance satisfying the fluctuation-dissipation theorem [40]: $\left\langle F(t) F\left(t^{\prime}\right)\right\rangle=k_{B} T \gamma\left(\left|t-t^{\prime}\right|\right)$. Note that the parameter $\gamma_{\alpha}$ describing viscosity is in units of $\mathrm{N} \mathrm{s}^{\alpha} / \mathrm{m}$.

Desposito and Vinales have derived the analytical formula for the limiting MSD [30] and then this formula was shown to be applicable to the time-averaged MSD for a long enough sample [31]. For sample length $N \delta t \gg \tau_{k}=$ $\left(\gamma_{\alpha} / k\right)^{1 / \alpha}$ and for small particles (when the inertia can be neglected), the exact equation takes the approximate form

$$
\left\langle\Delta r^{2}(t)\right\rangle=\frac{2 k_{B} T}{k} n\left(1-E_{\alpha}\left(-\frac{k}{\gamma_{\alpha}} t^{\alpha}\right)\right),
$$

where $E_{\alpha}$ is the Mittag-Leffler function. In the case of classical diffusion $(\alpha=1)$ eq. (3) simplifies to eq. (1).

The time $\tau_{k}=\left(\gamma_{\alpha} / k\right)^{1 / \alpha}$ characterizes the transition between the subdiffusive and confined regimes. At short times (i.e., $t \ll \tau_{k}$ ), one retrieves the subdiffusive scaling, $\left\langle\Delta r^{2}(t)\right\rangle \approx 2 n D_{\alpha} t^{\alpha} / \Gamma(1+\alpha)$, where $D_{\alpha}=k_{B} T / \gamma_{\alpha}$ is the generalized diffusion coefficient (in units of $\mathrm{m}^{2} / \mathrm{s}^{\alpha}$ ). In the opposite limit of long enough time (i.e., $\tau \gg \tau_{k}$ ), MSD slowly approaches a constant $2 k_{B} T / k$ set by the trapping potential. Two other time scales, $\tau_{m}=\sqrt{m / k}$ and $\tau_{p}=\left(\frac{m}{\gamma_{\alpha}}\right)^{\frac{1}{2-\alpha}}$ characterize the smallness of the inertial term in eq. (2) in comparison to the trapping and viscous forces, respectively. In appendix A we show that both of them are of the order of microseconds for our experiments, so that the inertia can indeed be neglected.

The complex shear modulus, $G^{*}=G^{\prime}+i G^{\prime \prime}$, is the quantity conventionally used in microrheology to represent the viscoelastic properties of the medium. Its real part, $G^{\prime}(\omega)$, is called elastic or storage modulus, and the imaginary part, $G^{\prime \prime}(\omega)$, is called viscous or loss modulus. The relationship between the MSD and the complex shear modulus is given by the Generalized Stokes-Einstein equation (GSE) $[7,41]$. The GSE equation allows one to obtain the Laplace transform of the viscoelastic shear modulus of the fluid from the Laplace transform of the MSD. In a trapping potential, the GSE equation reads [30]

$$
\tilde{G}(s)=\frac{k_{B} T}{\pi a s\left\langle\Delta r^{2}(s)\right\rangle}-\frac{k}{6 \pi a}-\frac{m s^{2}}{6 \pi a},
$$

where $\left\langle\Delta r^{2}(s)\right\rangle=\int_{0}^{\infty} \mathrm{d} t e^{-t s}\left\langle\Delta r^{2}(t)\right\rangle$ is a Laplace transform of the MSD, $\tilde{G}(s)$ is the Laplace transform of the stress relaxation modulus $G_{r}(t)$, which is related to complex shear modulus $G^{*}$ through the unilateral Fourier transformation.

The first term in eq. (4) is the classical GSE, the second term describes the influence of the OT, and the last term is the influence of the particles inertia which is negligible in most particle tracking experiments. Now, having the analytical eq. (3), we can obtain the analytical expression for complex shear modulus in the case of subdiffusion. Substituting eq. (3) into eq. (4) and following ref. [7] to replace the Laplace Transform with the Fourier Transform, we obtain the following expressions for viscoelastic moduli $G^{\prime}(\omega)$ and $G^{\prime \prime}(\omega)$ :

$$
\begin{aligned}
G^{\prime}(\omega) & =\frac{1}{6 \pi a} \gamma_{\alpha} \omega^{\alpha} \cos \left(\frac{\pi \alpha}{2}\right), \\
G^{\prime \prime}(\omega) & =\frac{1}{6 \pi a} \gamma_{\alpha} \omega^{\alpha} \sin \left(\frac{\pi \alpha}{2}\right) .
\end{aligned}
$$

This procedure is equivalent to the subtraction of the term $\frac{k}{6 \pi a}$ in eq. (4), where $k$ is the parameter, obtained from eq. (3). The expressions (5) are free from the influence of the trap and, thus, allow one to extend the region of analysis to the frequencies where traditional power law fitting is prevented by the trap. One therefore obtains the model-dependent continuation (eq. (5)) of $G^{\prime}$ to the lowfrequency range influenced by the trap. We expect eq. (5) to be correct for all frequencies corresponding to times at which eq. (3) fits well the experimental MSDs.

In this work, we are using eqs. (3) and (5) to calculate the viscoelastic moduli in the range where eq. (3) fits well, and the results of such calculation are compared with the results obtained by the program recently published in ref. [4].

\section{Results and discussion}

\subsection{MSD analysis for non-cancerous and cancerous cells}

Figure 2 presents the MSD of a typical dataset with the intermediate trap stiffness together with the corresponding MSD outside the cell. Datasets with other $k$ are given in the Supplementary Material (figs. 1SM-3SM). Different curves correspond to different granules trapped inside the cells (one granule per cell). At short times we observe the diffusive behavior in water and subdiffusion inside the cells. At long time the MSD becomes influenced by the optical potential and tends to a constant.

All curves were analyzed by fitting to eq. (3). The parameters of the fitting program have been defined as follows:

$$
X_{1}=\frac{2 k_{B} T}{k}, \quad X_{2}=\alpha, \quad X_{3}=\left(\frac{k}{\gamma_{\alpha}}\right)^{\frac{1}{\alpha}},
$$

$X_{1}$ is the empirical plateau value of the MSD curve which is defined by the trap stiffness, $X_{2}$ is the empirical subdiffusion exponent, and $X_{3}$ is the empirical crossover frequency, or the inverse of $\tau_{k}$ at which the subdiffusive motion starts to be confined. The parameters obtained from 10-12 different tracers for each cell line and each trap stiffness have been averaged and summarized in table 1 . The fitting interval has been chosen $10^{-5}-1 \mathrm{~s}$ which is 2 decades larger than the interval for which a power law fit would yield the same fitting quality. 
Table 1. Parameters obtained from fitting eq. (3) to the MSD of the granules inside cells. The uncertainty is the standard deviation of parameters between different granules.

\begin{tabular}{|c|c|c|c|c|}
\hline Trap stiffness $(\mathrm{N} / \mathrm{m})$ & Cell line & $X_{1}\left(\mathrm{~nm}^{2}\right)$ & $X_{2}$ & $X_{3}(\mathrm{~Hz})$ \\
\hline \multirow{2}{*}{$4 \cdot 10^{-6}$} & HCV29 & $1277 \pm 721$ & $0.70 \pm 0.07$ & $11 \pm 9$ \\
\cline { 2 - 5 } & T24 & $1413 \pm 669$ & $0.69 \pm 0.07$ & $6 \pm 3$ \\
\hline \multirow{2}{*}{$10^{-5}$} & HCV29 & $679 \pm 258$ & $0.73 \pm 0.02$ & $36 \pm 18$ \\
\cline { 2 - 5 } & T24 & $487 \pm 198$ & $0.67 \pm 0.05$ & $10 \pm 7$ \\
\hline \multirow{2}{*}{$3 \cdot 10^{-5}$} & HCV29 & $186 \pm 58$ & $0.73 \pm 0.04$ & $172 \pm 88$ \\
\cline { 2 - 5 } & T24 & $218 \pm 52$ & $0.75 \pm 0.03$ & $166 \pm 103$ \\
\hline
\end{tabular}

The simple power law fit fails for all lag times greater than $10^{-2} \mathrm{~s}$ (see fig. 4SM). The subdiffusion coefficients obtained from the fit by means of eq. (3) and by the power law fit for different fitting intervals are given in table 2SM together with the error variance of the fit.

One can easily observe that the subdiffusion exponent, $X_{2}$, is the same for cancerous and non-cancerous cells within the experimental uncertainty. Its value is close to $3 / 4$, the value predicted by the theory of semiflexible polymers. This result confirms the findings reported for the mammalian cells in refs. $[9,20,24,26]$ and contradicts the recent ref. [27] for yeast cells.

The absolute values of MSD and the plateau parameter $X_{1}$ have to be taken with caution because of two reasons: the experimental uncertainty coming from the same calibration applied to granules of slightly different sizes, and the wide distribution of the MSD (see figs. 1SM-3SM). The wide distribution of MSD in the semiflexible networks has been observed both in vitro [42] and in vivo [9,20] and has been attributed to the high heterogeneity of the network.

The most important difference between the cell lines is observed in the crossover frequency, $X_{3}$, which is smaller for the cancerous cells in two datasets. It corresponds to the passage to the confined regime at longer times (bigger $\left.\tau_{k}\right)$. In the case of the highest trap stiffness the difference is insignificant confirming that the observed effect originates from the low-frequency properties of the cells.

In order to compare the long-time behavior of the MSDs of the two cell lines visually, the MSD curves have been normalized to make their values coincide at the minimal lag time. This procedure is justified by the fact that for short times, $\left\langle\Delta r^{2}(t)\right\rangle \approx \frac{2 k_{B} T}{\gamma_{\alpha} \Gamma(1+\alpha)} t^{\alpha}$, and thus it should not depend on the trap stiffness. The resulting curves are presented in fig. 3 which shows the difference in the long lag time behavior of MSD for the two cell lines. For all three datasets, the MSDs of the cancerous cells exceed the MSDs of the non-cancerous cells at $t>\tau_{k}$. This result agrees with the data of ref. [43] in which fig. 2 shows that at the lag time about $0.05 \mathrm{~s}$ (initial point in ref. [43]) the MSDs of the non-cancerous and cancerous cells approach each other; whereas, at longer times the MSD of the cancerous cells increases more rapidly than MSD of the non-cancerous ones.

The possible explanations for the bigger MSD of the cancerous cells are numerous. Most importantly, it corresponds to the lower stiffness at low frequency observed

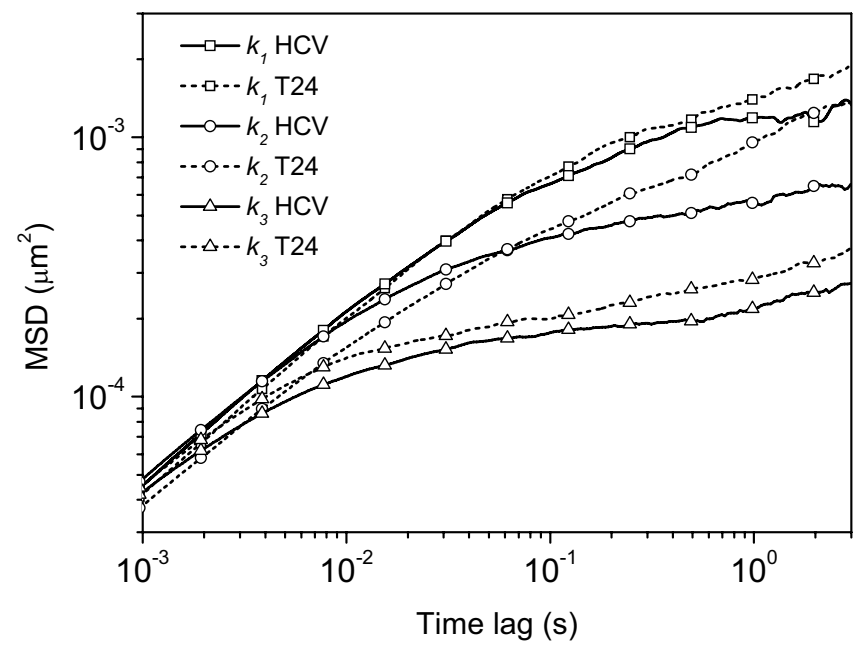

Fig. 3. Ensemble-averaged and renormalized MSD for the noncancerous (full lines) and cancerous (dashed lines) cells for different trap stiffness. The MSD of the cancerous cells exceeds the MSD of the non-cancerous cells for all trap powers at lag times $\tau>\tau_{k}$.

for these cells in AFM $[28,29]$. Another important consideration is the impact of molecular motors and other types of metabolic activity. As previously noted, the number of the tracers escaping the trap during recording was higher for the T24 cells. References $[12,26,44]$ have shown that the molecular motors generate strong random fluctuations particularly apparent at time scales longer than $0.1 \mathrm{~s}$. The resulting random displacements have a superdiffusive character which is usually explained by the nonthermal forces $[8,45]$. The passage from subdiffusion to superdiffusion has been shown in refs. [22,46-48] and others. In our case the superdiffusion would be hindered by the trap, but it may produce the effective increase of $\tau_{k}$. If the bigger $\tau_{k}$ in cancerous cells is the consequence of superdiffusion, it means that the molecular motors are more active in the cancerous cells. For example, blocking the myosin II motors by blebbistatin results in the decrease of long-time MSD, and the effect is more pronounced in the cancerous cells, as illustrated in the Supplementary Material (fig. 5SM). The detailed discussion of this topic will be a subject of a separate publication.

Note that for the strongest trap the difference in $\tau_{k}$ between cancerous and non-cancerous cells is insignificant because the strong confinement overcomes the motor ac- 
tivity almost completely. In fact, in order to overcome the trap force, several motors should work collectively [49], and the probability of such collective action becomes too small for the bigger force.

The short-time $\left(10^{-5}-10^{-3} \mathrm{~s}\right)$ MSDs of both cell lines coincide within experimental uncertainty. If we extrapolate the unconstrained (without influence of the trap) MSD as $\frac{2 k_{B} T}{\gamma_{\alpha} \Gamma(1+\alpha)} t^{\alpha}$ using the parameters from table 1, we obtain MSD at $0.05 \mathrm{~s}$ equal $10^{-3} \mu \mathrm{m}^{2}$ that corresponds closely to the values from ref. [43] at $0.05 \mathrm{~s}$ for both cancerous and non-cancerous cells. In order to compare our results to the MSDs reported in refs. [20,26], the MSD dependence on the tracer size (MSD $\sim a^{-1}$ ) has to be considered. We prefer thus to compare the viscoelastic moduli, for which tracer size is already taken into account.

\subsection{Calculation of viscoelastic moduli $G^{\prime}(\omega)$ and $\mathrm{G}^{\prime \prime}(\omega)$}

We have calculated the viscoelastic moduli from the experimental MSDs using the procedure described recently in [4]. This procedure makes use of the relationship between the complex shear modulus $G^{*}(\omega)$ and the Fourier transformed compliance $\hat{J}(\omega)$ and allows one to avoid the Laplace transform. It correctly preserves the experimental noise and requires a single theoretical parameter: the inverse of the gradient of MSD at the long time limit. As we suppose that the MSD in the trap at long time tends to a constant, we have used the high value for its inversed gradient $\left(\eta=10^{10} \mathrm{Pas}\right)$ that provides the constant value of $G^{\prime}(\omega)$ at low frequency limit. We have observed that increasing further this parameter had almost no influence on the results. In the following we call the moduli obtained by this procedure the experimental moduli.

Figure 4 compares the experimental moduli to the model-dependent ones calculated using eq. (5) with parameters of table 1 for the strongest trap and the weakest traps. The low-frequency parts of the experimental curves are noisy due to the small number of points for the calculation. The model-dependent curves fit the experimental ones for the high frequency - this is the direct consequence of the corresponding fit of MSD at small times. At low frequencies the addition of a trap-dependent constant $G_{\text {trap }}^{\prime}=\frac{2 k_{B} T}{6 \pi a X_{1}}$ to $G^{\prime}(\omega)$ from eq. (5) is required to make the two curves superimpose. Figure 4 also shows the model-dependent elastic modulus $G^{\prime}(\omega)$ without the influence of the trap which we consider justified as long as the fit of the trap-dependent curve is good $(\omega>10 \mathrm{~Hz})$. Despite of the fact that the influence of the trap is significant even for the weakest trap, eq. (5) allows us to extract the model-dependent moduli for the region $10-10^{5} \mathrm{~Hz}$.

The model-dependent moduli without the influence of the trap for all tracers of T24 have been averaged to obtain fig. 5a. The geometric mean has been used to keep the correct power law, and the results are presented in the log scale. After the subtraction of the trap influence the viscoelastic moduli almost coincide within the statistical uncertainty for all six datasets: two cell types and three

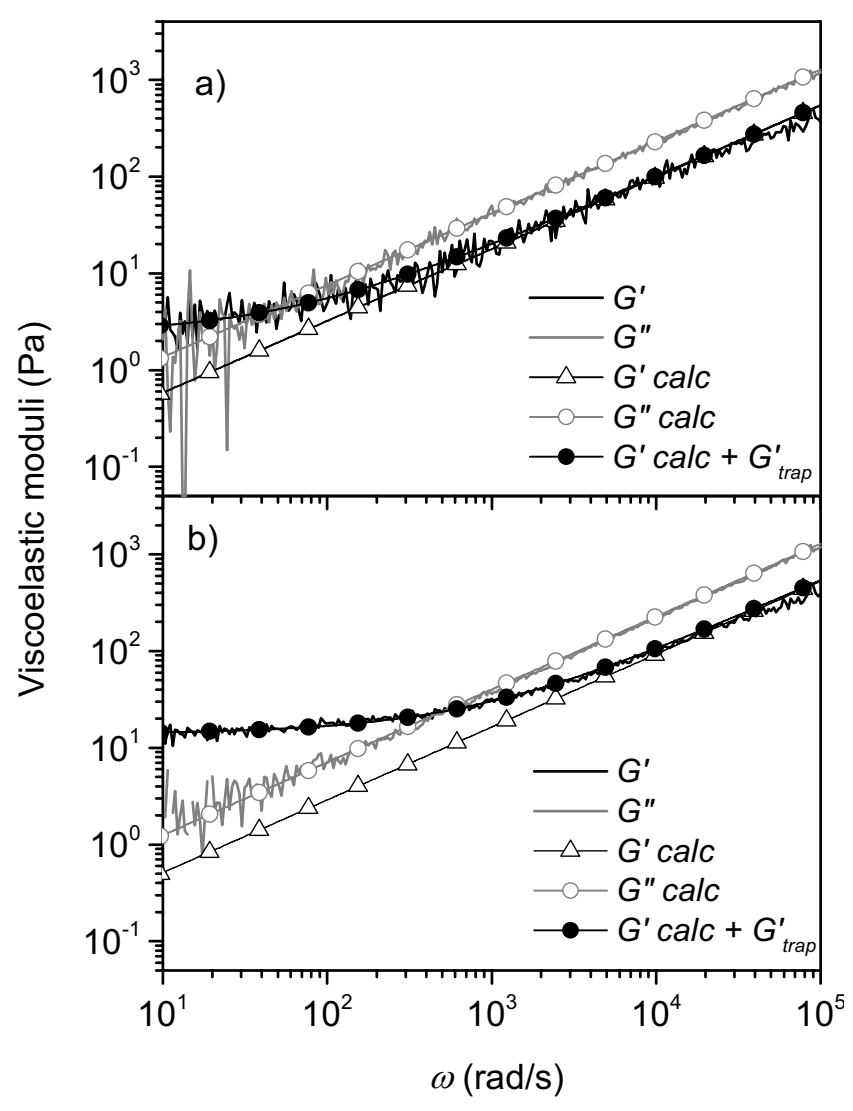

Fig. 4. Frequency dependence of the viscoelastic moduli, $G^{\prime}$ and $G^{\prime \prime}$, for the non-cancerous HCV29 cells obtained with trapping spring constants a) $k_{1}$ and b) $k_{3}$. The noisy traces are the experimental moduli obtained by the program of ref. [4], and the calculated curves are obtained using eq. (5). The experimental storage modulus is affected by the trapping spring constant, and after its subtraction as described in the text the moduli become the same for different traps.

values of stiffness. The high statistical uncertainty is the consequence of the wide distribution of the MSD values, and the moduli below $50 \mathrm{~Hz}$ are not significant as the standard deviation exceeded the mean value (see fig. 5a).

Figure 5b provides the summary of our results for the significant region of $10^{2}-10^{5} \mathrm{~Hz}$ for both cell lines calculated as the geometric mean over the moduli obtained with different trap forces. The difference between the cell lines is insignificant within the experimental error. One can notice that the viscous modulus is generally larger than the elastic one. It is a direct consequence of the slope $3 / 4$ because the ratio of the viscoelastic moduli is $G^{\prime \prime} / G^{\prime}=\tan (\pi \alpha / 2)$.

The comparison with literature data is complicated by the fact that the more traditional particle tracking microrheology usually gives the viscoelastic moduli for frequencies $0.01-10 \mathrm{~Hz}$. We have found only two works in which the high-frequency intracellular viscoelastic moduli for the living cells are provided: ref. [24] for human kidney and refs. [20,26] for monkey kidney TC7 epithelial cell lines. The moduli of ref. [24] are about $5-7$ times bigger than ours at frequencies $10^{3}-10^{5} \mathrm{~Hz}$. The moduli 

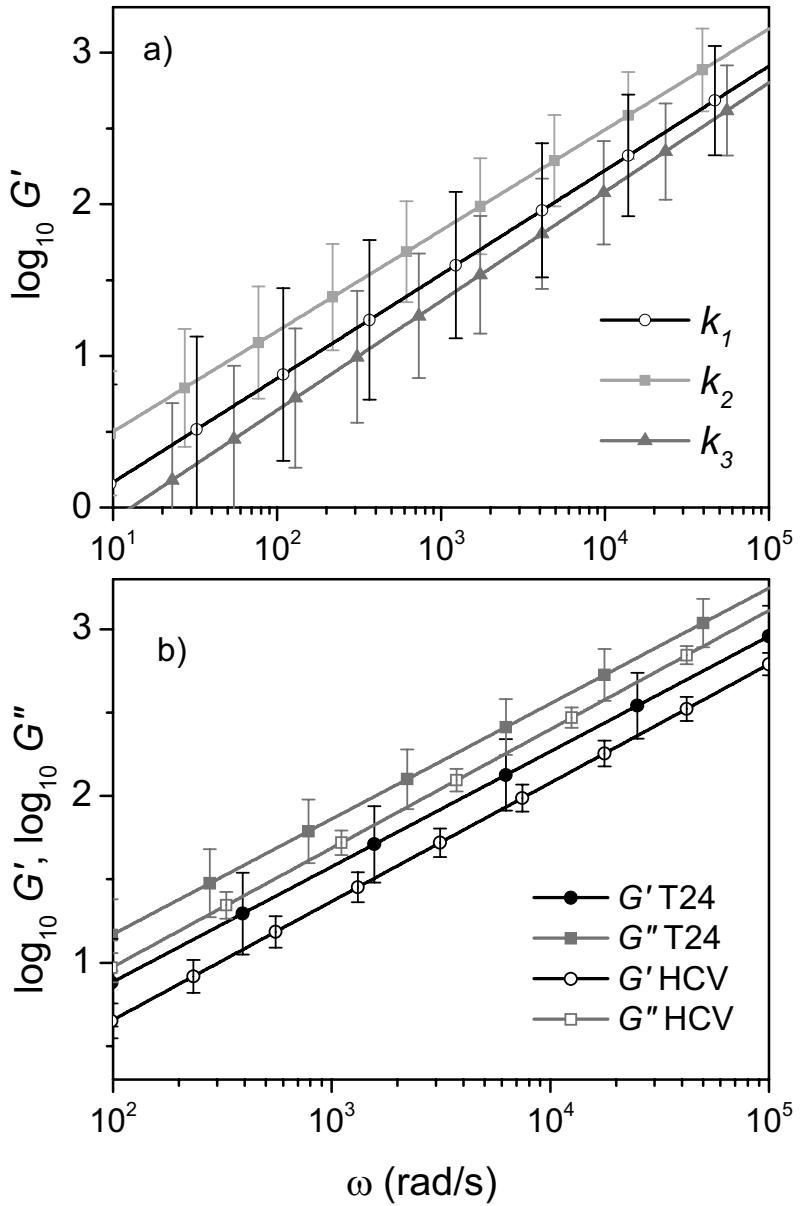

Fig. 5. Model-dependent viscoelastic moduli, $\log _{10}(G(\mathrm{~Pa}))$, calculated using eq. (5) from different datasets. (a) elastic modulus, $G^{\prime}$, for experiments in T24 cell line with different trap spring constant. The error bars are the SD between the different tracers within one spring constant dataset. (b) Viscoelastic moduli, $G^{\prime}$, (black) and $G^{\prime \prime}$ (grey) for the two cell lines. For each cell line the viscous modulus is bigger than the elastic modulus. The error bars are the SD between the different datasets. The difference between the cell lines is insignificant within the experimental error.

presented in refs $[20,26]$ are consistent with the ones that we obtain for the high-frequency region. Notably, at the frequency $1000 \mathrm{rad} / \mathrm{s}$, ref. [26] gives $\left|G^{*}\right|=40 \mathrm{~Pa}$ against our $44 \mathrm{~Pa}$.

As already mentioned in the MSD analysis, ref. [43] provides the MSD similar to our results for breast epithelial MCF-7 (cancerous) and MCF-10A (non-cancerous) cells with the tracers of $0.5 \mu \mathrm{m}$ diameter, but lacks the high-frequency region. Reference [43] does not provide the viscoelastic moduli obtained from the MSD, but discusses that they are of the order of several pascals; whereas, the moduli obtained for the same cells with AFM are of the order of $\mathrm{kPa}$. It is exactly the same situation for the cell lines studied in this work. The AFM values for HCV29 and T24 cells reported in ref. [28] are: $G^{\prime}=10.3 \pm 3.4 \mathrm{kPa}$ for HCV29 and $G^{\prime}=1.8 \pm 0.6 \mathrm{kPa}$ for T24 - orders of magnitude larger than our results at low frequencies.
In agreement with ref. [43] this shows that the intracellular microrheological data cannot be directly compared to the AFM stiffness measurements. The two possible reasons for this difference are: i) the AFM elastic modulus is obtained in the quasi-static regime, and the cell has time to react, ii) the microrheology probes the dynamics of the cytoskeleton itself; whereas, the AFM probably probes a combination of the elastic shell and the viscoelastic interior of the cell. Therefore, this confirms the idea that the difference between the cancerous and non-cancerous cells is not due to their cytoskeleton structure.

\section{Conclusions}

We have measured the mean square displacements of the endogenous tracers in the non-cancerous HCV29 and cancerous T24 epithelial cells of human urine bladder and tested the new theoretical approach proposed in refs. [30, 31]. Both HCV29 and T24 cells reveal close sub-diffusive exponents: $0.72 \pm 0.04$ for HCV29 and $0.70 \pm 0.04$ for T24 which seem to correspond to the prediction of the model of semi-flexible polymers [18]. The viscoelastic moduli have been calculated in the model-dependent manner and provide the reasonable values in the region $10^{2}-10^{5} \mathrm{~Hz}$ where the model of semiflexible polymers well describes tracer motion.

We have not found significant difference between the HCV29 and T24 cells at the frequencies $10^{2}-10^{5} \mathrm{~Hz}$, which suggests that the cytoskeleton polymer network of the two cell lines has similar structure. Nevertheless, we have observed a tendency of the cancerous cells to show the larger MSDs at long lag time, confirming the earlier findings in the other microrheological studies of cancerous and noncancerous cells [43].

The increase in MSD shows that at low frequencies the cancerous cells possess the ability to restructure the cytoskeleton to adapt to the external force. This provides a possible explanation for the AFM results [28,29] which had shown an order of magnitude difference in the stiffness of these cell lines.

The authors thank Dr. Manlio Tassieri for the Matlab code and useful comments, Dr. Malgorzata Lekka for providing the cell lines, and Dr. Janusz Lekki for the technical support in the instrumental part. Elena Bertseva thanks for support the National Swiss Fond MHV subsidy PMPDP2_134178/1. We also thank Dr. Andrzej Kulik and Chiara Gabella for useful discussions.

\section{Appendix A.}

The generalized Langevin equation (2) has three characteristic times:

$\tau_{m}=\sqrt{m / k}$ : time of crossover from ballistic to trapconfined motion,

$\tau_{p}=\left(\frac{m}{\gamma_{\alpha}}\right)^{\frac{1}{2-\alpha}}$ : time of crossover from ballistic to diffusive motion,

$\tau_{k}=\left(\gamma_{\alpha} / k\right)^{1 / \alpha}$ : time of crossover from diffusive to trap-confined motion. 
For a bead of radius $0.2 \mu \mathrm{m}$ and a density close to the one of water (as all our tracers have such density), the mass $m$ is about $3 \cdot 10^{-17} \mathrm{~kg}$. Taking the intermediate trap stiffness $k=10^{-5} \mathrm{~N} / \mathrm{m}$, we obtain $\tau_{m}=\sqrt{m / k}$ about $2 \cdot 10^{-6} \mathrm{~s}$. On the other hand, the time of crossover from diffusive to trap-confined motion, $\tau_{k}=\left(\gamma_{\alpha} / k\right)^{1 / \alpha}$, is obtained from the fitting of experimental MSDs, and is bigger than $5 \cdot 10^{-3} \mathrm{~s}$ (the value for the strongest trap). The time limit of ballistic regime, $\tau_{p}$, can be expressed through the first two, and equals

$$
\tau_{p}=\left(\frac{m}{\gamma_{\alpha}}\right)^{\frac{1}{2-\alpha}}=\left(\frac{\tau_{m}^{2}}{\tau_{k}^{\alpha}}\right)^{\frac{1}{2-\alpha}} \approx 10^{-7} \mathrm{~s} .
$$

As the lower time limit of our investigation is $10^{-5} \mathrm{~s}$, the inertia of the particle has been neglected in the solution of eq. (2)

\section{References}

1. A. Yao et al., Lab. Chip 9, 2568 (2009).

2. Y. Kimura, J. Phys. Soc. Jpn. 78, 041005 (2009).

3. D. Wirtz, Annu. Rev. Biophys. 38, 301 (2009).

4. R.M.L. Evans et al., Phys. Rev. E 80, 012501 (2009).

5. C. Guzman et al., Appl. Phys. Lett. 93, 184102 (2008).

6. H. Lee et al., Acta Biomateri. 6, 1207 (2010).

7. T.G. Mason, Rheol. Acta 39, 371 (2000).

8. P. Bursac et al., Nat. Mater. 4, 557 (2005).

9. L. Deng et al., Nat. Mater. 5, 636 (2006).

10. M.-T. Wei et al., Opt. Express 16, 8594 (2008).

11. L.A. Hough, H.D. Ou-Yang, Phys. Rev. E 73, 031802 (2006).

12. D. Mizuno et al., Macromolecules 41, 7194 (2008).

13. D. Raucher et al., in Methods in Cell Biology (Academic Press, 2008) pp. 451.

14. S. Suresh, Acta Mater. 55, 3989 (2007).

15. K.E. Kasza et al., Curr. Opin. Cell Biol. 19, 101 (2007).

16. M.R.K. Mofrad, Annu. Rev. Fluid Mech. 41, 433 (2009).
17. Y.-C. Lin et al., Soft Matter 7, 902 (2011).

18. F. Gittes, F.C. MacKintosh, Phys. Rev. E 58, R1241 (1998).

19. D.C. Morse, Macromolecules 31, 7044 (1998).

20. K.M. Van Citters et al., Biophys. J. 91, 3946 (2006).

21. C.M. Hale et al., PLoS ONE 4, e7054 (2009).

22. L. Bruno et al., Phys. Rev. E 80, 011912 (2009).

23. M.L. Gardel et al., Phys. Rev. Lett. 93, 188102 (2004).

24. S. Yamada et al., Biophys. J. 78, 1736 (2000).

25. I.M. Tolic-Norrelykke et al., Phys. Rev. Lett. 93, 078102 (2004).

26. B.D. Hoffman et al., Proc. Natl. Acad. Sci. U.S.A. 103, 10259 (2006).

27. J.-H. Jeon et al., Phys. Rev. Lett. 106, 048103 (2011).

28. M. Lekka et al., Biochim. Biophys. Acta 1540, 127 (2001).

29. M. Lekka et al., Eur. Biophys. J. 28, 312 (1999).

30. M.A. Desposito, A.D. Vinales, Phys. Rev. E 80, 021111 (2009).

31. D. Grebenkov, Phys. Rev. E 83, 061117 (2011).

32. E. Bertseva et al., Nanotechnology 20, 285709 (2009).

33. S. Jeney et al., Nanotechnology 21, 255102 (2010).

34. K.C. Neuman, S.M. Block, Rev. Sci. Instrum. 75, 2787 (2004).

35. A. Rohrbach et al., Rev. Sci. Instrum. 75, 2197 (2004).

36. S. Jeney et al., Phys. Rev. Lett. 100, 240604 (2008).

37. F. Gittes, G. Schmidt, Opt. Lett. 23, 7 (1998).

38. M.J. Saxton, K. Jacobson, Annu. Rev. Biophys. Biomol. Struct. 26, 373 (1997).

39. S. Chandrasekhar, Rev. Mod. Phys. 15, 1 (1943).

40. R. Kubo et al., Statistical Physics II. Nonequilibrium Statistical Mechanics (Springer, Berlin, 1985).

41. T.G. Mason et al., J. Opt. Soc. Am. A 14, 139 (1997).

42. T. Gisler, D.A. Weitz, Phys. Rev. Lett. 82, 1606 (1999).

43. Y. Li et al., J. Biomed. Opt. 14, 064005 (2009).

44. D. Mizuno et al., Science 315, 370 (2007).

45. A.W.C. Lau et al., Phys. Rev. Lett. 91, 198101 (2003).

46. Y. Tseng et al., Biophys. J. 83, 3162 (2002).

47. M.A. Despósito et al., Physica A 390, 1026 (2011).

48. D. Arcizet et al., Phys. Rev. Lett. 101, 248103 (2008).

49. A. Caspi et al., Phys. Rev. E 66, 011916 (2002). 